\title{
NEAREST NORMAL APPROXIMATION FOR CERTAIN OPERATORS
}

\author{
JOHN PHILLIPS
}

\begin{abstract}
In this paper we show that binormal operators have nearest normal approximants. In fact, we exhibit nearest normals to such operators and, as a corollary, show that the hermitian part of a binormal operator with real spectrum is a nearest normal. We obtain further corollaries on nearest normal approximation to operators which are square roots of normal operators and then apply these results to perturbations of operator algebras.
\end{abstract}

Introduction. In this paper we show that binormal operators have nearest normal approximants. In fact, we exhibit nearest normals to such operators and, as a corollary, show that the hermitian part of a binormal operator with real spectrum is a nearest normal. We obtain further corollaries on nearest normal approximation to operators which are square roots of normal operators and then apply these results to perturbations of operator algebras.

1. The main theorem. If $\Lambda$ is a (closed) subset of the complex numbers and $\Re(\Lambda)$ denotes the set of all normal operators on some fixed Hilbert space $\mathcal{H}$ with spectrum contained in $\Lambda$, then for any operator, $A$, on $\mathcal{H}$ one can pose a number of questions. For example:

(1) Is $\inf \{\|B-A\|: B$ in $\Re(\Lambda)\}$ attained?

(2) If so, what is an example of such a $B$ ?

(3) Is there a "formula" for $\inf \{\|B-A\|: B$ in $\Re(\Lambda)\}$ in terms of $A$ ?

If $A$ is a normal operator and $\Lambda$ is a closed set then Halmos answered these questions in [5]. In the case $\Lambda$ is the unit circle, these questions are essentially answered in [3] and [9]. For $\Lambda=[0, \infty)$ see [4].

In this section, we put $\Lambda$ equal to the set of complex numbers, and for the class of binormal operators we answer questions (1) and (2) and in a weak sense question (3).

1.1. THEOREM. Let $N$ be a binormal operator whose upper triangular form is

$$
N=\left[\begin{array}{cc}
A & B \\
0 & C
\end{array}\right] \quad(\text { see }[8, \text { Theorem 7.20]). }
$$

Then,

Received by the editors November 29, 1976.

AMS (MOS) subject classifications (1970). Primary 47A30, 47B20, 47C15; Secondary 46L05, $46 \mathrm{~L} 10$.

Key words and phrases. Normal operator, binormal operator, normal approximation, perturbations of operator algebras.

C American Mathematical Society 1978 
(1) $\inf \{\|N-T\|: T$ is normal $\}=\frac{1}{2}\|B\|$,

$$
\left[\begin{array}{cc}
A & \frac{1}{2} B \\
\frac{1}{2} V^{2} B^{*} & C
\end{array}\right] \text { is a nearest normal to } N
$$

where $(A-C)=V|A-C|$ and $V$ is unitary.

Proof By perturbing $N$ slightly we first assume that $A, B$, and $C$ are diagonal. That is,

$$
A=\left[\begin{array}{ccc}
a_{1} & & 0 \\
& \ddots & \\
0 & & a_{n}
\end{array}\right], \quad B=\left[\begin{array}{lll}
b_{1} & & 0 \\
& \ddots & \\
0 & & b_{n}
\end{array}\right], \quad C=\left[\begin{array}{lll}
c_{1} & & 0 \\
& \ddots & \\
0 & & c_{n}
\end{array}\right],
$$

where $n$ is some ordinal number. Moreover, we assume $\left|b_{1}\right|=\|B\|$.

Now, let $X$ be any normal operator; then in matrix form $X=\left(x_{i j}\right)$, where $i, j$ run from 1 to $2 n$. Thus, if $z_{j}$ denotes the $j$ th column vector of $(N-X)$, we have

$$
\begin{aligned}
\|N-X\|^{2} & \geqslant \max \left\{\left\|z_{1}\right\|^{2},\left\|z_{n+1}\right\|^{2}\right\} \\
& >\max \left\{\left|a_{1}-x_{11}\right|^{2}+\sum_{k=2}^{2 n}\left|x_{k 1}\right|^{2},\left|b_{1}-x_{1 n+1}\right|^{2}\right\} \\
& =\max \left\{\left|a_{1}-x_{11}\right|^{2}+\sum_{k=2}^{2 n}\left|x_{1 k}\right|^{2},\left|b_{1}-x_{1 n+1}\right|^{2}\right\} \\
& >\max \left\{\left|x_{1 n+1}\right|^{2},\left|b_{1}-x_{1 n+1}\right|^{2}\right\},
\end{aligned}
$$

where $\sum_{k=1}^{2 n}\left|x_{\mathbf{k} 1}\right|^{2}=\sum_{k=1}^{2 n}\left|x_{1 k}\right|^{2}$ because $X$ is normal. Hence,

$$
\begin{aligned}
\|N-X\| & \geqslant \max \left\{\left|x_{1 n+1}\right|,\left|b_{1}-x_{1 n+1}\right|\right\} \\
& \geqslant \frac{1}{2}\left\{\left|x_{1 n+1}\right|+\left|b_{1}-x_{n+1}\right|\right\}>\frac{1}{2}\left|b_{1}\right|=\frac{1}{2}\|B\| .
\end{aligned}
$$

By continuity we have that $\|N-X\|>\frac{1}{2}\|B\|$ even if $A, B$, and $C$ are not diagonal.

Now let $V$ be a unitary operator commuting with $A, B$, and $C$ such that $(A-C)=V|A-C|$. It is an easy calculation that the operator

$$
X_{0}=\left[\begin{array}{cc}
A & \frac{1}{2} B \\
\frac{1}{2} V^{2} B^{*} & C
\end{array}\right]
$$

is normal. Clearly, $\left\|N-X_{0}\right\|=\frac{1}{2}\|B\|$ so that the theorem is proved.

1.2. CoRollary. If $N$ is binormal and has real spectrum, then the hermitian part of $N$ is a nearest normal to $N$.

Proof. If $N$ has real spectrum, then $N=\left[\begin{array}{cc}A & B \\ 0\end{array}\right]$ where $A$ and $C$ are hermitian. Clearly 


$$
\frac{1}{2}\left(N+N^{*}\right)=\left[\begin{array}{cc}
A & \frac{1}{2} B \\
\frac{1}{2} B^{*} & C
\end{array}\right]
$$

is of distance $\frac{1}{2}\|B\|$ from $N$ and, therefore, by Theorem 1.1, is a nearest normal to $N$.

REMARK. The converse to 1.2 is false as can be seen by very simple examples. However, for $2 \times 2$ complex matrices the converse is true.

1.3. Remark. As noted by the referee, Theorem 1.1 implies that for $N$ a binormal operator,

$$
\operatorname{distance}(N, \text { normal operators }) \leqslant \frac{1}{2}\left\|N^{*} N-N N^{*}\right\|^{1 / 2},
$$

and that this estimate is sharp for $N=\left[\begin{array}{ll}0 & 1 \\ 0 & 0\end{array}\right]$.

For $n$-normal operators, by first reducing to the $n \times n$ matrix case and then by putting the matrix in upper triangular form, one can show that approximating $N$ by its diagonal yields the very crude estimate:

$$
\text { distance }\left(N \text {, normal operators) } \leqslant(n-1)\left\|N^{*} N-N N^{*}\right\|^{1 / 2}\right. \text {. }
$$

Clearly, this estimate is not sharp and it would be interesting to know what the best estimate is.

2. Square roots of normal operators. By [7, Theorem 1], if $N$ is the square root of a normal operator, then $N=\left[\begin{array}{ll}B & C \\ 0 & -B\end{array}\right] \oplus A$, where $A$ and $B$ are normal and $C$ is a positive one-to-one operator commuting with $B$. It is easy to see that the technique of Theorem 1.1 shows that the normal operator

$$
\left[\begin{array}{cc}
B & \frac{1}{2} C \\
\frac{1}{2} V^{2} C & -B
\end{array}\right] \oplus A \quad \text { is a nearest normal to } N
$$

where $B=V|B|$ and $V$ is unitary. Specializing to the case $N^{2}=0$, so that $N=\left[\begin{array}{ll}0 & C \\ 0 & 0\end{array}\right] \oplus \mathrm{O}$ with $C \geqslant 0$, we see that

$$
\left[\begin{array}{cc}
0 & \frac{1}{2} C \\
\frac{1}{2} C & 0
\end{array}\right] \oplus 0
$$

is a nearest normal to $N$, which is of distance $\frac{1}{2}\|N\|$ from $N$. Although this is trivial to prove if $N$ is a complex $2 \times 2$ matrix, the general case, $N^{2}=0$, seems to require a little more work.

3. Perturbations of operator algebras. Let $\mathcal{Q}$ and $\mathscr{B}$ be $C^{*}$-algebras on the Hilbert space $\mathscr{H}$. For simplicity, we usually assume that $\mathscr{Q}$ and $\mathscr{B}$ each act essentially on $\mathscr{H}$ (i.e. $I \in \mathbb{Q}^{-} \cap \mathscr{B}^{-}$where ${ }^{-}$denotes weak-operator closure). Following Kadison and Kastler we define $\|\mathscr{Q}-\mathscr{B}\|$ by

$$
\|Q-\mathscr{B}\|=\sup \left\{\left\|A-\mathscr{B}_{1}\right\|,\left\|B-\mathbb{Q}_{1}\right\|: A \text { in } \mathbb{Q}_{1}, B \text { in } \mathscr{B}_{1}\right\}
$$

where $\mathscr{C}_{1}, \mathscr{B}_{1}$ are the unit balls of $\mathscr{Q}$ and $\mathscr{B}$, respectively.

The following proposition is an improvement on the best published result ( $k=\frac{1}{4}$ in [1]) and the best unpublished result known to the author, $k=\frac{1}{3}$, due to H. Behnke. 
3.1. Proposition. Let $\mathfrak{Q}$ and $\mathfrak{B}$ be $C^{*}$-algebras on $\mathcal{H}$ (not necessarily acting essentially) and suppose $\|\mathbb{Q}-\mathscr{B}\|<k\left(\leqslant \frac{1}{2}\right)$ then $\mathbb{Q}$ is abelian if and only if $\mathscr{B}$ is abelian.

Proof. By Lemma 5 of $[6]$ we can assume that $\mathscr{Q}$ and $\mathscr{B}$ are weak-operator closed. Suppose that $\mathscr{Q}$ is abelian and $\mathscr{B}$ is not. By the structure theory for von Neumann algebras, $\mathscr{B}$ contains a copy of the 2-by-2 matrices, and therefore an operator of the form $N^{2}=0,\|N\|=1$. By the example of $\$ 2$, the nearest normal to $N$ is of distance $\frac{1}{2}$ from $N$. Hence, $\|\mathscr{Q}-\mathscr{B}\| \geqslant \frac{1}{2}$.

3.2. Lemma. If $\|\mathscr{Q}-\mathscr{B}\|<k(\leqslant 1)$ and $E$ is a projection in $\mathcal{Q}$, then there is a projection $F$ in $\mathscr{B}$ with $\|E-F\|<k(\leqslant 1)$.

Proof. Since $\mathbb{Q}$ acts essentially, $U=(I-2 E)$ is a unitary in $\mathbb{Q}$, so there is a $V$ in $\mathscr{B}_{1}$ such that $\|U-V\|<k$. Then, if $X=\frac{1}{2} I-\frac{1}{2} V,\|X\| \leqslant 1$ and $\|E-X\|<\frac{1}{2} k$. Also, if $Y=\frac{1}{2}\left(X+X^{*}\right)$ then $\|E-Y\|<\frac{1}{2} k$ and $Y$ is selfadjoint. If we now proceed as in the proof of Lemma 2.1 of [1], there is a projection $F$ in $\mathscr{B}$ with $\|E-F\|<k$.

3.3. Corollary. If $\mathscr{Q}$ and $\mathscr{B}$ are von Neumann algebras, with $\mathcal{Q}$ abelian and $\|\mathfrak{Q}-\mathscr{B}\|<k\left(\leqslant \frac{1}{2}\right)$, then there is exactly one isomorphism $\Phi$ of $\mathscr{Q}$ onto $\mathscr{B}$ such that $\sup _{\|A\|<1}\|\Phi(A)-A\|<k$.

Proof. Use Proposition 3.1 to conclude that $\mathscr{B}$ is abelian and then use Lemma 3.2 to conclude that the projection lattices of $\mathcal{Q}$ and $\mathscr{B}$ are of distance less than $k$ from each other. Now, apply Lemma 3.1 of [1] to obtain the result.

3.4. Corollary. If $\mathscr{Q}$ and $\mathfrak{B}$ are von Neumann algebras, with $\mathscr{Q}$ abelian and $\|\mathscr{Q}-\mathscr{B}\|<k\left(\leqslant \frac{1}{2}\right)$, then there is a unitary $W$ in $(\mathscr{Q} \cup \mathfrak{B})^{\prime \prime}$ such that $\mathscr{B}=W \mathfrak{Q} W^{*}$ and

$$
\|I-W\| \leqslant 2^{1 / 2} k\left(1+\left(1-k^{2}\right)^{1 / 2}\right)^{-1 / 2} .
$$

Proof. Use Corollary 3.3 and then Proposition 4.2 of [2] to obtain the conclusion.

ACKNOWLEDGEMENT. The author would like to thank the referee for his/ her helpful comments and suggestions.

\section{REFERENCES}

1. E. Christensen, Perturbations of type I von Neumann algebras, J. London Math. Soc. 9 (1975), 395-405.

2. __ Perturbations of operator algebras, Univ. of Oslo preprint series, no. 14, 1974.

3. K. Fan and A. J. Hoffman, Some metric inequalities in the space of matrices, Proc. Amer. Math. Soc. 6(1955), 111-116.

4. P. R. Halmos, Positive approximants of operators, Indiana Univ. Math. J. 21 (1972), 951-960. 5. 51-58. Spectral approximants of normal operators, Proc. Edinburgh Math. Soc. 19 (1974),

6. R. V. Kadison and D. Kastler, Perturbations of von Neumann algebras. I: Stability of type, Amer. J. Math. 94 (1972), 38-54. 
7. H. Radjavi and P. Rosenthal, On roots of normal operators, J. Math. Anal. Appl. 34 (1971), $653-664$.

8. ___ Inoariant subspaces, Springer-Verlag, New York, 1973.

9. D. J. van Riemsdijk, Some metric inequalities in the space of bounded linear operators on a separable Hibert space, Nieuw Arch. Wisk. (3) 20 (1972), 216-230.

Dapartmient of Mathimatics, Dahousie University, Haltix, Nova Scotin Canada 